\title{
Design an Optimum PV System for the Satellite Technology using High Efficiency Solar Cells
}

\author{
Ahmed Lotfy \\ Faculty of Engineering \\ Ain Shams University \\ Cairo, Egypt
}

\author{
Wagdy R. Anis \\ Professor \\ Faculty of Engineering \\ Ain Shams University \\ Cairo, Egypt
}

\author{
M. A. Atalla \\ Professor \\ Alexandria Higher Institute of \\ Engineering and Technology \\ Alexandria, Egypt
}

\author{
Joseph V. M. Halim \\ Professor \\ Faculty of Engineering \\ Ain Shams University \\ Cairo, Egypt
}

\author{
M. Abouelatta \\ Associate Professor \\ Faculty of Engineering \\ Ain Shams University \\ Cairo, Egypt
}

\begin{abstract}
This paper presents an optimum design of a photovoltaic (PV) power system for the satellite market using high efficiency solar cells. This is to enhance the power capabilities of the satellite solar arrays without extra challenges in area, weight and budget constraints. In this way, the designed power system can efficiently fulfill the power requirements of the satellite subsystems, either in daylight or eclipse periods. In this paper, the presented design is applied on GEO satellites, for which the solar arrays use triple junction cells technology. The required area and mass of the solar array are calculated. This is in addition to estimating the required number of assembled cells in the designed solar array. The optimum size of the satellite batteries, used in eclipse periods, is also determined. Finally, an economic analysis is performed to demonstrate the financial budget of the satellite project.
\end{abstract}

\section{Keywords}

Multijunction Solar cell, GEO, Satellite Orbit, Batteries, Design PV, Efficiency, Economics.

\section{INTRODUCTION}

In the current modern era, artificial satellites are considered as one of the greatest mankind creations. Satellites are used for several services and applications like broadcasting satellite services (BSS), fixed satellite services (FSS), remote sensing and earth observation services, meteorological and navigation services, search and rescue operations in addition to the space telescopes and the military purposes.

Satellite orbits are greatly varying, depending on the required mission of the satellite, and they are classified into a number of ways. In this paper, the designed PV system will be applied on Geostationary Earth Orbit (GEO) satellites.

Satellites are usually constructed from semi-independent computer-controlled subsystems, which attend many tasks such as power generation, thermal control, telemetry and command, attitude control and orbital control.

The power generation source is always one of the most sensitive and complicated issue in the field of satellite science due to several issues:

- The challenge of the area limitation constraints for the solar panels versus the required power capacity forces the satellite technology to develop solar cells with high efficiencies.

- High advanced technology for batteries are required to efficiently supply the satellite subsystems during the periodic eclipse durations. This is in addition to fulfill the requirement of batteries long lifetime due to the impossibility of their replacement and maintenance.

- The cost of the main components is very high, which lead to many theoretical and technical researches for decreasing the financial budget while preserving the same technical requirements.

Photovoltaic solar arrays are the prime way of converting the solar energy to an efficiently electrical energy for satellites. Array configurations depend on the spacecraft stabilization concept, the orbit type, and the power requirements. For example, as the power requirements of the three-axis stabilization spacecraft increase, it is expected that the trend will be directed to have an extendible solar array. The single crystal silicon cell is the basic building block of the currently solar arrays manufacturing. However, the thin-film solar cells, like Gallium Arsenide (GaAs) and triple junction (3J) solar cells, can offer several extra technical advantages.

In this paper, an optimum design for the satellite solar arrays will be presented and discussed with several technical considerations. The design can be summarized in the following steps:

- Defining the satellite orbital period and the eclipse duration, used in the presented design.

- Identifying the power demand of the satellite subsystems and determining the corresponding power capacity required from the solar PV power system.

- Identifying the optimum solar cell type to fit with the available solar panels area and the power demands.

- Estimating the required area and mass of the satellite solar array, using the selected solar cell type, in addition to calculating the corresponding total number of assembled solar cells that can be used in the solar array. 
- Calculating the optimum sizing of the battery bank storage and selecting the type of batteries that copes with the satellite lifetime and the available space and weight.

This paper is organized as follows: Section 2 clarifies the concept of the solar irradiance variations for the satellite throughout the year. The details of the solar array design are discussed in section 3 . In sections 4 , the sizing of the batteries is calculated. The economic analysis is presented in section 5 . Finally, the conclusion is drawn in section 6.

\section{SOLAR IRRADIANCE}

The variation in the earth-sun distance, due to the elliptical path of the earth around the sun, results in about $\pm 3 \%$ in the extraterrestrial solar flux. The formula that expresses the extraterrestrial solar radiation intensity $G_{s c}$ is given by [1]

$G_{s c}=G_{s c o}\left(1+0.033 \cos \frac{360 d}{365}\right) \mathrm{kw} / \mathrm{m}^{2}$

where $\mathrm{d}$ is the order of the day $(1 \leq \mathrm{d} \leq 365)$ and $G_{s c o}$ is the solar constant $\left(1.35 \mathrm{kw} / \mathrm{m}^{2}\right)$. The variation in the $G_{s c}$ during a complete year is shown in Fig.1.

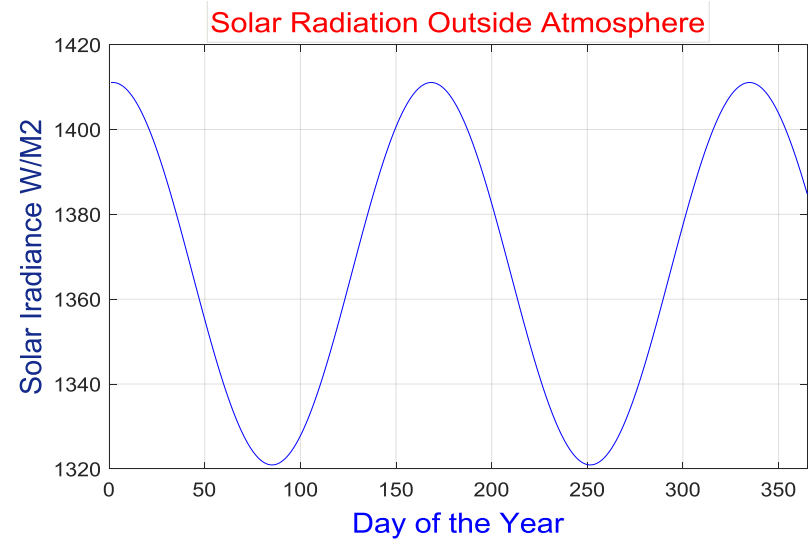

Fig.1 Ratio of extraterrestrial irradiance at normal incidence to solar constant

\section{SOLAR ARRAY DESIGN}

For earth orbiting satellites, the photovoltaic solar arrays must be designed so that they can fulfill the power requirements of the spacecraft subsystems through both daylight and eclipse periods [2].

In this paper, a geostationary satellite of 20 years lifetime, $4000 \mathrm{~kg}$ total mass, 23.56 orbital period and maximum eclipse duration of 72 minutes [3] will be used as a study case. In the presented design, the power generation system will use the following data.

- The required average power is assumed to be $7000 \mathrm{~W}$ either during daylight or eclipse, $P e=P_{d}=7000 \mathrm{~W}$.

- It is assumed that a power tracking mechanism is applied by using a Solar Array Drive Mechanism (SADM), to have a peak generated power during daylight.

The efficiency of the satellite power subsystem will be roughly $X_{d}=0.8$ and $X_{e}=0.6$ where $X_{d}$ is the efficiency of the spacecraft power subsystem to supply the power from the array to the loads in daylight. However, $X_{e}$ is the efficiency of the spacecraft power subsystem to supply the power from the array to the batteries for charging during daylight and from the batteries to the loads during eclipse [4].
- The degradation per year for the multi-junction solar cells, like the triple junctions (3J) cells, is $0.5 \%$ whereas it is $2.75 \%$ for GaAs solar cells [5]. Also, the nominal value of the inherent degradation (Id) is 0.77[6].

In this paper, the solar array design will follow the following steps:

1. Selection of the solar cells type:

Triple junction $3 \mathrm{~J}(\mathrm{GaInP} / \mathrm{GaInAs} / \mathrm{Ge})$ cells are recommended for both LEO and GEO satellites since they have higher efficiency than GaAs cells. AlthougFh the cost of the 3J cells is higher than GaAs, they are smaller and lighter, comparing to GaAs cells. This presents big funds for the launching costs, while satisfying the same power capability of the larger and heavier GaAs arrays.

2. Determining the solar array power capability:

The total extent of the generated power, that can be achieved by the solar array during the non-shadowing periods, is determined by summing the power requirements of the spacecraft subsystems during daylight and the needed power to charge the secondary batteries to fulfill the eclipse power demands. The solar array generated power $P_{s a}$ can be expressed as:

$P_{s a}=\frac{\frac{P_{e} T_{e}}{X_{e}}+\frac{P_{d} T_{d}}{X_{d}}}{T_{d}}=9.34 \mathrm{k}$ Watt

where $P_{e}$ and $P_{d}$ represent the spacecraft power requirements during eclipse and daylight, respectively. $T_{e}$ and $T_{d}$ are the eclipse and the daylight durations for the satellite in its orbit, respectively.

3. Estimate the output power per unit area of the solar array:

The output power per unit area $P_{o}$ can be calculated by multiplying the efficiency of the PV material ( $\eta$ ) by the solar radiation intensity $\left(G_{s c}\right)$. Since the $3 \mathrm{~J}$ cells power efficiency is $30.7 \%$ and using the worst case of the solar radiation intensity $G_{s c}=1321 \mathrm{w} / \mathrm{m}^{2}, P_{o}$ can be presented as:

$P_{o}=$ solar intensity $\times \eta=405.547 \mathrm{Watt} / \mathrm{m}^{2}$

4. Estimate the required output power at the beginning of life (BOL) of the satellite:

The required power at the beginning of life is given by:

$P_{\text {Bol }}=P_{o} I_{d} \cos \theta=312.27$ Watt

where $\theta$ is the incidence angle between the vector normal to the solar arrays and the direction of the sun line $\left(\theta=23.5^{\circ}\right.$ in worst case) [7]. In the presented design, $\theta$ equals 0 since the satellite uses a power tracking mechanism.

5. Estimate the output power at the satellite end of life (EOL):

The power degradation factor $\left(L_{d}\right)$ through the satellite lifetime is calculated as:

$L_{d}=(1-\text { degradation per year })^{\text {satellite life }}=0.9$

As mention, before the degradation per year for $3 \mathrm{~J}$ solar cell is $0.5 \%$. Therefore, the output power at the satellite EOL can be estimated as:

$P_{\text {Eol }}=P_{\text {Bol }} L_{d}=281.043 \mathrm{Watt}$ 
Fig.2 shows the degradation in the delivered output power of the power subsystem throughout the lifetime of the satellite. As shown in the figure, the performance of the solar panels using $3 \mathrm{~J}$ solar cells is better than that can be achieved using the GaAs solar cells due to the higher efficiency of the $3 \mathrm{~J}$ cells.

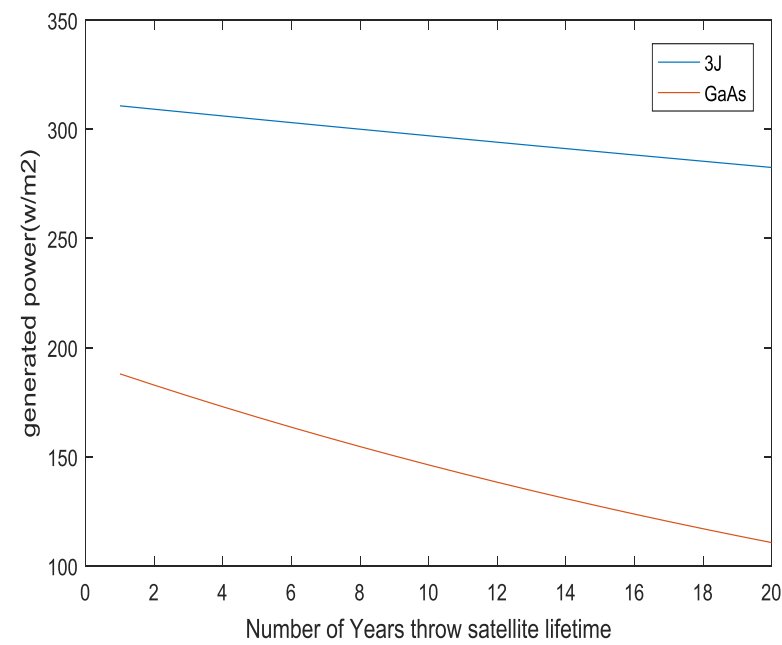

Fig. 2 Output power degradation over the satellite lifetime

Determining the required area and mass of the solar array: The solar array area can be estimated as:

$A_{s a}=\frac{P_{s a}}{P_{E o l}}=33.23 \mathrm{~m}^{2}$

The final step is to estimate the mass of the solar array using equation (8), where the specific performance of the planar array is taken to be $70 \mathrm{~W} / \mathrm{Kg}$ [8]

$M_{a}=\left(\frac{1}{70}\right) P_{s a}=133.43 \mathrm{~kg}$

The data of the Spectrolab for the 3J solar cells [9], can be used in the presented design, where the Bus voltage is $V_{B u s}=100 v d c$, the cell power is $P_{c e l l}=3.493 \mathrm{w}$, the cell current is $I_{\text {cell }}=17.4 \mathrm{~mA} / \mathrm{cm}^{2}$ and the cell voltage is $V_{\text {cell }}=$ 2.390 v. Using the calculated power of the solar array, $P_{s a}=9.34 \mathrm{~K} \mathrm{Watt}$, the total number of the triple (3J) cells, required to be assembled in the solar array, can be obtained as follows:

$\mathrm{N}=\frac{P_{\text {Sa }}}{P_{\text {Cell }}}=2673.92 \cong 2674$

For comparison, the pervious calculations are performed in case of using GaAs cells in the satellite solar array. The used data for GaAs cells is given in the Spectrolab [10], where the Bus voltage is $V_{B u s}=100 v d c$, the cell power is $P_{\text {cell }}=$ $1.2593 \mathrm{w}$, the cell current is $I_{\text {cell }}=28.6 \mathrm{~mA} / \mathrm{cm}^{2}$ and the cell voltage is $V_{\text {cell }}=0.900 \mathrm{v}$. The calculated results for the GaAs cells show that the solar array area $A_{s a}$ is $84.79 \mathrm{~m}^{2}$, the solar array mass $M_{a}$ is $373.6 \mathrm{~kg}$ and the total number of the GaAs assembled cells, required in the solar array, is 7417 cells.

As shown in the results, the used $3 \mathrm{~J}$ solar cells can fulfill the same satellite power requirements using lighter mass, smaller area and lower number of assembled cells, compared to the GaAs cells.

\section{BATTERY SIZING}

Batteries are a basic part of the satellite power subsystem. Spacecraft's use the generated power of the solar arrays, during the non-shadowing periods to charge the batteries.
During the eclipse periods, the batteries use their energy storage to perform the role of the solar arrays and providing the satellite subsystems with their required power.

The steps for calculating the battery sizing will be presented in the following steps:

1. Selecting the type of the secondary batteries:

There are many types of the batteries that can be used in satellite manufacturing such as Nickel Cadmium (NiCd), Nickel Hydrogen, Lithium Ion, and sodium Sulfur. However, Li-ion batteries are still the common storage devices for various satellite applications. This is because they have been efficiently qualified for the space technology and there is a wide database for this battery type in most missions.

2. Determine the number of cells needed in the battery:

The required data, used to calculate the batteries size, is given in [11], where the cell voltage is $V_{\text {cell }}=3.6 \mathrm{v}$, the Depth of Discharge (DOD) is assumed to be $80 \%$ and the energy density $E_{d}$ is $150 \mathrm{Wh} / \mathrm{Kg}$ for (Li-ion VES180), which is the used battery type in this paper.as Mentioned before, that the calculated power of the solar array is $P_{s a}=9.34 \mathrm{~K} \mathrm{Watt}$, the eclipse duration is 72 minutes and the bus voltage is $V_{B u s}=$ $100 \mathrm{Vdc}$. Therefore, the number of cells $(\mathrm{N})$, needed in the battery, can be calculated as:

$N=\frac{V_{\text {bus }}}{V_{\text {cell }}}=33.33 \cong 33$ cells

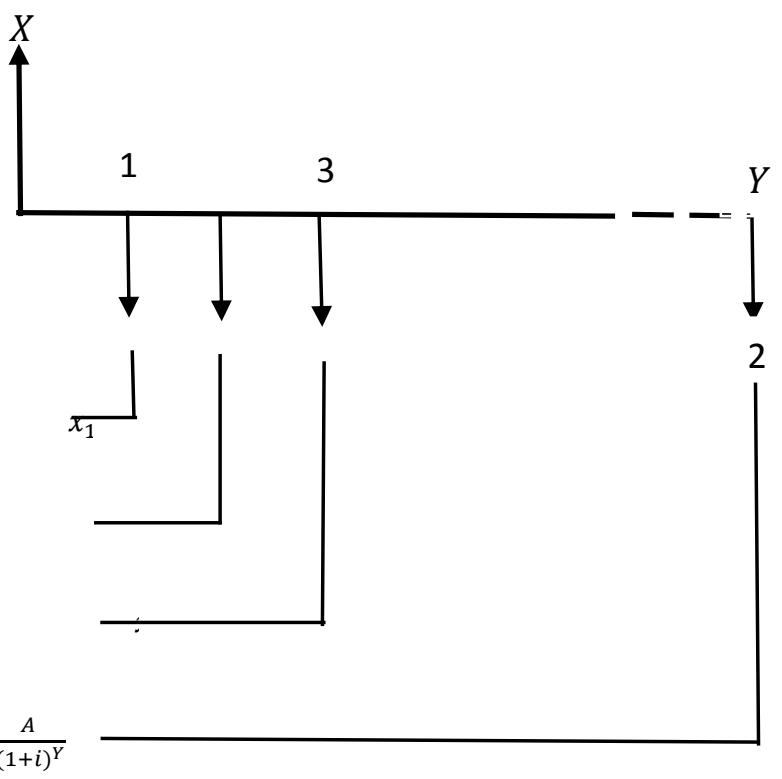

Fig. 3 payment flow

To avoid overloading, the bus voltage $V_{B}$ can be expressed as:

$V_{B}=N \times V_{\text {cell }}=118.8 \mathrm{Vdc}$

3. Calculating the total required storage capacity of the battery:

The total capacity $(C)$ of the battery is given as in [12].

$C=\frac{\left(P_{s a}\right)(\text { max eclipse duration })}{(\text { depth of discharge })\left(V_{B}\right)}$

$=117.93 \mathrm{Ah}$

This yields to a battery required storage capacity, $C_{B}$ as follows: 
$C_{B}=C \times V_{B}=14.01 \mathrm{KWh}$

4. Estimate total mass of Batteries required during eclipse.

The last step is to estimate the total mass of the batteries, required to power the spacecraft subsystems during eclipse. This can be calculated as shown in the following equation.

$M_{\text {Batt }}=\frac{C_{B}}{E_{d}}=93.4 \mathrm{Kg}$

For comparison, the pervious calculations are performed in case of using NiCd batteries [13]. The calculated results of the $\mathrm{NiCd}$ batteries show that the capacity and the mass of the batteries are $22.416 \mathrm{kWh}$ and $747.2 \mathrm{~kg}$, respectively.

As shown in the results, the selected Li-ion batteries can fulfill the same storage power requirements using lower mass and capacity constraints, compared to the $\mathrm{NiCd}$ batteries.

\section{ECONOMIC ANALYSIS}

There are various ways to perform the economic analysis for the satellite market. In this paper, the present value analysis method will be applied.

In the economic present value analysis, there are two approaches of payment; uniform and geometric gradient payments. This paper will follow the uniform approach, where all the installments of payment are equal. Denote $(X)$ as the initial payment, (A) is the installment amount of payment, (i) is the annual interest rate and $(Y)$ is the number of annual installments, which is considered to be equal to the satellite life time [14].

If an amount $(X)$ is borrowed from a bank and the annual installment payment is $(A)$, then the present value $(x)$ of the amount $(A)$ after $(Y)$ years will be represented as in equation (15) and fig 3.

$x=\frac{A}{(1+i)^{Y}}$

It should be noted that $(y)$ is the year number throughout the satellite lifetime, where $1 \leq y \leq Y$.

Therefore, the present value $(X)$ of the annual payments after $(Y)$ successive years is expressed by:

$X=\sum_{y=1}^{Y} x=\frac{A}{1+i}+\frac{A}{(1+i)^{2}}+\frac{A}{(1+i)^{3}}+\cdots+\frac{A}{(1+i)^{Y}}$

By multiplying both sides by $(1+\mathrm{i})$, this yields to:

$X(1+i)=A+\frac{A}{1+i}+\frac{A}{(1+i)^{2}}+\frac{A}{(1+i)^{3}}+\cdots+\frac{A}{(1+i)^{Y-1}}$

By subtracting Equation (16) from Equation (17), the value of (X) can be represented as:

$X=A *\left(\frac{1-(1+i)^{-Y}}{i}\right)$

Equation (18) is only valid when the following conditions are satisfied; the first installment is paid after one year from the initial payment $(X)$, the amount of the installments are equal and the durations between the periodic installments are also equal, as in case of the annual installments.

Finally, the total cost $\left(C_{t}\right)$ of the satellite, including the launch and manufacturing costs, can be expressed as:

$C_{t}=M C R_{0}\left[\frac{1-(1+i)^{-Y}}{i}\right]$

Noting that the parameter, which the launching cost depends on, is presented in [15] where $R_{0}$ is the annual rent per uplinked channel to the satellite transponders, $M$ is the total number of channels and $C$ is the occupancy rate, which represents the percentage of the rented channels with respect to the total available number of the channels that can be supported by the satellite transponders. Also, it should be noted that the value (M C $\left.R_{o}\right)$ expresses the annual income of the satellite project.

The following Figures represent the analytical results of the relation between the annual rent per channel $\left(R_{o}\right)$ in Million dollars, the percentage of the occupancy rate $(C)$ and the percentage of the annual interest rate (i). The Figures are expressed under the following parameters; the satellite has a life time of 20 years, 950 channels and a total cost, including the launch and manufacturing costs, 370 million dollars.

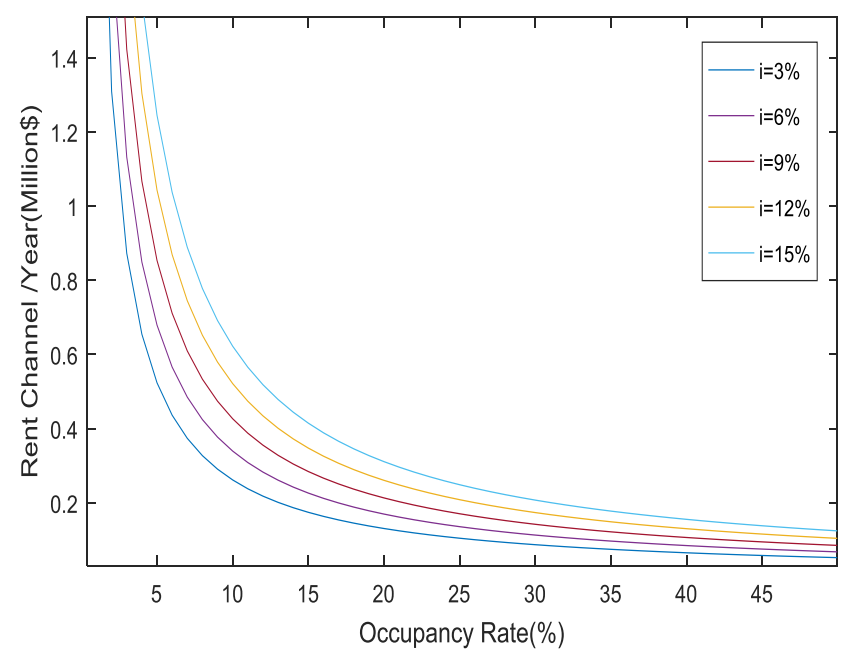

Fig. 4: The occupancy rate versus the yearly renting payment per channel under various values of annual interest rates.

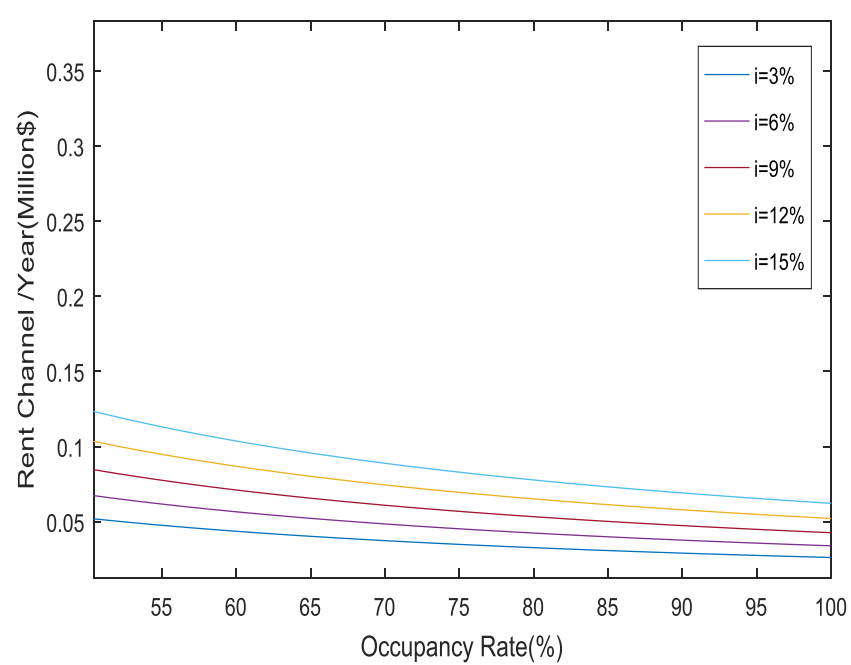

Fig. 5: The occupancy rate (in the range between $45 \%$ and $100 \%$ ) versus the yearly renting payment per channel under various values of annual interest rates.

Figures 4 and 5 illustrate the relation between the occupancy rate and the yearly renting payment per channel for various values of interest rates. As shown in the figures, the occupancy rate is inversely proportional with the yearly renting payment per channel. This is because as the percentage of the already rented channels increases, the offered yearly payment for channel renting decreases. It should be noted that the rate of change in the channel renting 
payment is fast until approximately $25 \%$ occupancy rate and it is significantly decreases after this percentage until reaching approximately constant value. In economically point of view, this is reasonable since for low occupancy rates, the channel renting payment is needed to be high. However, as the occupancy rates increase, the channel renting payment becomes low till reaching a constant value level that achieves a considerable revenue to the satellite owner. To clarify the results in the range of $45 \%$ to $100 \%$ of the occupancy rates, Figure 5 zooms the results of the yearly renting payment per channel versus the occupancy rates under various values of annual interest rates.

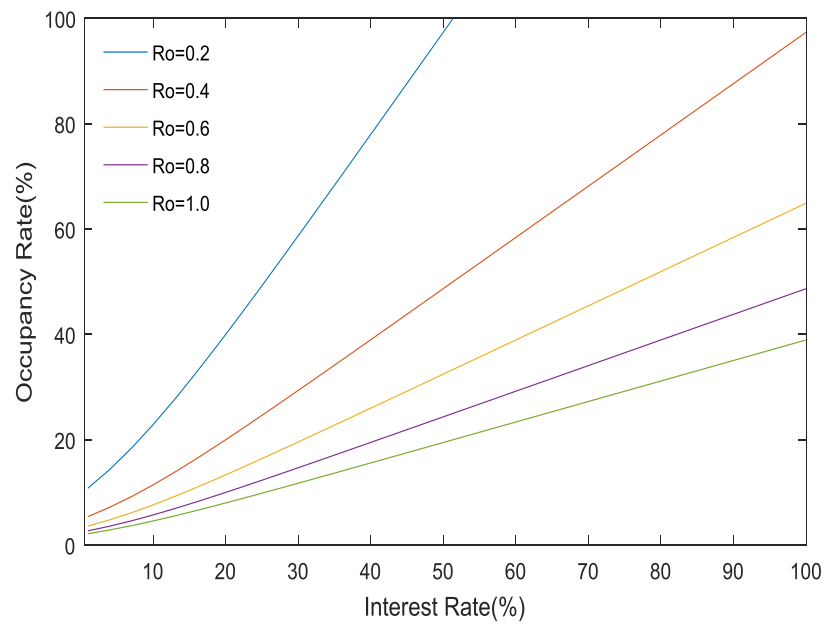

Fig. 6: The increase effect of the applied interest rate on the required occupancy rate for different values of channel annual renting payment.

Figure 6 presents the relation between the required occupancy rate and the applied interest rate under different values of channel annual renting payment. The figure shows a directly proportional relation between the required occupancy rate and the applied interest rate for the same value of channel renting payment. This is because as the applied interest rate increases, the required number of channels, needed to be rent, increases, respectively. Also, for the same interest rate, as the value of channel annual renting payment increases, the possibility to have a high percentage of the occupancy rate decreases.

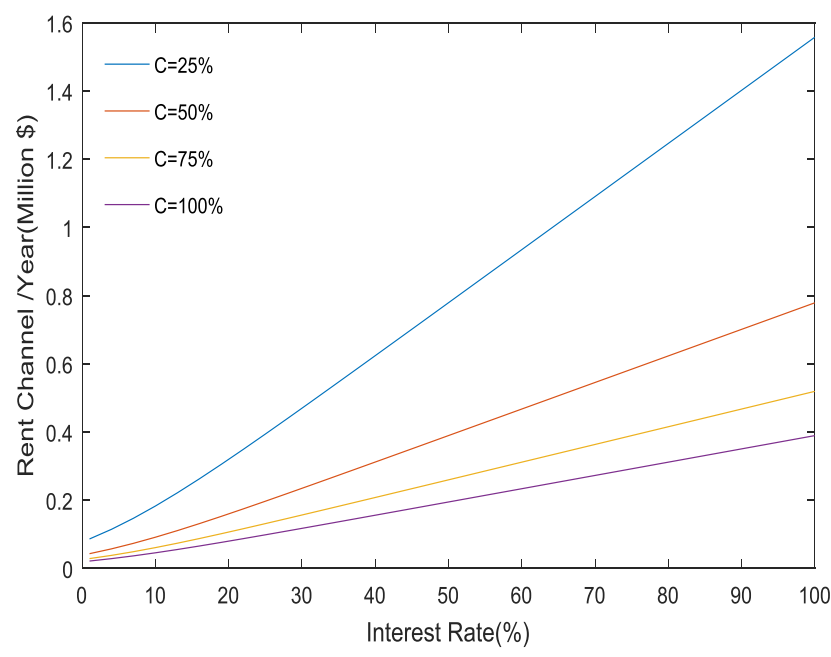

Fig. 7: The relation between the applied interest rate and the estimated amount of the channel renting payment.
Figure 7 illustrates the effect of the applied interest rate on the estimated amount of the channel renting payment, required to have a considerable revenue to the satellite owner, As shown in the figure, the channel renting payment should be increased as the applied interest rate increases. Moreover, the graph draws also the same deduction of Figure 5 since the renting payment decreases as the occupancy rate increases.

\section{CONCLUSION}

In this paper, an optimum design of a photovoltaic power system for the satellite technology is presented. The key feature in the design lies in its capability to enhance the required satellite power during daylight and eclipse, while profitably reducing the area, weight and budget constraints. The design takes the advantages of both the triple junction solar cells technology for manufacturing the solar arrays and the Lithium-ion batteries for storage. For the same required power, the numerical results show that the used triple junction solar cells can achieve significant technical advantages over the GaAs solar cells, which are the currently most-common type in satellite manufacturing market. This is due to the higher performance of the triple junction cell and its capability to efficiently generate an extra power, owing to its higher efficiency. Consequently, the triple junction cells can significantly reduce the required manufacturing parameters of the solar arrays including area, weight and number of assembled cells. Also, the numerical results illustrate that the Lithium-ion batteries outperform the NiCd batteries in both the required storage capacity and the total mass. Referring to the previous results, the combination between the triple junction solar cells and the Lithium-ion batteries in the presented design can offer big funds to the financial budget of the satellite project. Finally, the uniform approach of the economic present value analysis is applied to estimate the financial budget of the satellite project itself. The economical results demonstrate an interesting behavior between the yearly renting payment per channel, the occupancy rate and the annual interest rate. The curves draw that the channel renting payment decreases as the occupancy rate increases. However, the required occupancy rate is directly proportional with the applied interest rate. Moreover, the estimated amount of the channel renting payment, required to have a considerable revenue to the satellite owner, should be increased as the applied interest rate increases.

\section{REFERENCES}

[1] Donald Rapp. "Solar Energy" . Prentice-Hall, Inc.,pp. 53, 1981.

[2] Larson, Wiley J., and James Richard Wertz. "Space mission analysis and design". Microcosm, Inc., Torrance, CA (US) third edition, pp. 411, 1999.

[3] Brown, Charles D. "Elements of spacecraft design". Aiaa, 2002, pp.326

[4] Larson, Wiley J., and James Richard Wertz. "Space mission analysis and design". Microcosm, Inc., Torrance, CA (US) third edition, pp. 413, 1999.

[5] Larson, Wiley J., and James Richard Wertz. "Space mission analysis and design". Microcosm, Inc., Torrance, CA (US) third edition, pp. 412, 1999.

[6] Larson, Wiley J., and James Richard Wertz. "Space mission analysis and design". Microcosm, Inc., Torrance, CA (US) third edition, pp. 414, 1999. 
[7] Larson, Wiley J., and James Richard Wertz. "Space mission analysis and design". Microcosm, Inc., Torrance, CA (US) third edition, pp. 417, 1999.

[8] Donald Rapp, Tom Hamilton, "Solar Cell and Array Technology for Future Space Science Missions". California institute of technology Pasadena, California, pp. 8, 2002.

[9] 30.7\% Triple Junction Space Grade Solar Cell spectrolab XTJ prime triple junction solar cell.pdf.

[10] GaAs/Ge Single Junction Solar Cells http://www.spectrolab.com/DataSheets/SJCell/sj.pdf
[11] Saft-space "Saft batteries... powering outer space for 50 years: extreme performance batteries meeting the demands of space applications", www.saftbatteries .com

[12] Oraby, Osama A., et al. "UASat Solar Array Design and Performance Characteristics". 2014.

[13] Larson, Wiley J., and James Richard Wertz. "Space mission analysis and design". Microcosm, Inc., Torrance, CA (US) third edition, pp. 420, 1999.

[14] L.Blank and A. Tarquin "Basic of Engineering Economics" McGraw Hill, New York, pp.29,2008.

[15] 1st Symposium on Space Educational Activities, December 9-12, 2015, Padova, Italy. 